\title{
Los límites de la profesión de ser profesor
}

The limits of the profession of being professor

Robinson Roa Acosta y Édgar Orlay Valbuena Ussa

Universidad Pedagógica Nacional. Departamento de Biología. Línea de Investigación Conocimiento Profesional del Profesor de Ciencias. robinsonroa@hotmail.com, edgarorlay@hotmail.com

\section{Resumen}

En este artículo se presentan y analizan algunos elementos que directa o indirectamente configuran la imagen de la profesión de profesor y que limitan o delimitan su quehacer. Se formulan algunas preguntas abiertas que aproximan al panorama general y ayudan a vislumbrar la situación de los espejismos que ocultan posicionamientos políticos y económicos, dejando de lado el reconocimiento del saber pedagógico y con él la degradación de la identidad y autonomía profesional del profesor.

\section{Palabras claves}

Profesión de profesor, imagen, autonomía, saber pedagógico.

\section{Introducción}

La imagen que sobre los profesores y su profesión tienen ellos mismos y la sociedad en general, tiene un gran espectro de variables e implicaciones tanto para la misma profesión como para el desarrollo social de la educación, sociedad, economía, ciencia y tecnología, así como también, para controlar la tendencia de gobiemo e ideología. Dicha imagen tiene mucho que ver con el contexto en el cual "se ejerce y desarrolla la profesión" y con quienes se interactúa, al igual que, prioritariamente, con las normas que regulan el sistema educativo.

En tal sentido, gran parte de la profesión de profesor está regulada desde el exterior a la misma profesión, es decir, los reaursos de infraestructura física, monetaria, didácticos, salarios, formación inicial y permanente del profesorado, logros alcanzados por los estudiantes, están en manos del estado, careciendo de participación el profesorado. La toma de decisiones del profesor se encuentras enmarcadas en el desarrollo y gestión curricular, que pierden sentido al finalizar el proceso educativo.

Para efecto de desarrollar algunos de estos aspectos que en su conjunto constituyen un intrincado sistema de elementos que configuran la imagen de la profesión del profesor, como eje articulador y visible ante los resultados en educación, se pretende aproximar a la situación de su profesión, fundamentalmente describiendo ¿Quiénes?, ¿Por qué? y ¿para qué ingresan a la actividad de la enseñanza? y ¿Cuál es el poder de decisión del profesor frente a la promoción de los estudiantes? Se presentan y analizan algunos ejemplos que ilustran de primera mano los límites de la profesión en el contexto nacional. 


\section{Primer acercamiento al panorama de la profesión de profesor}

Algunas de las preguntas que surgen entorno a esto, que permiten iniciar a configurar la idea sobre el panorama de la profesión del profesor, no se plantean para contestarlas puntualmente ni para desarrollar minuciosamente en este escrito, sino más bien para aproximarse a ellas, son las siguientes: ¿De qué profesor estamos hablando y para qué sociedad? ¿Cuál es la imagen de profesor que las condiciones y situaciones permiten formar? ¿Por qué la profesión de profesor es y ha sido tan manejada desde el exterior a su actividad en el aula de dase? ¿Existe algo particular en relación con el manejo que los gobiemos le dan a profesión? ¿Quiénes son lo que por lo general acceden a ser profesores profesionales o profesores, y por qué? ¿Qué conocimiento y saber especializado debe conformar la profesión del profesor para ser profesor?

"Una de las ideas más extendidas en la actualidad con respecto al profesorado $y$, a la vez, una de las más polémicas es su condición de profesional. Ya sea como expresión de una aspiración, como descripción de las características del oficio de enseñar, o como discusión sobre las peculiaridades o limitaciones con que tal condición se da en los docentes, el tema del profesionalismo parece bastante instalado en el discurso teónico, así como en las expresiones de los propios docentes sobre su trabajo" (Contreras, 1997: 17).

Ante esto se pueden señalar que: es común escuchar que enseñar es fácil y que por lo tanto el ejercicio de profesor lo puede realizar la persona que medianamente crea lo puede hacer; se habla sobre la autonomía de los profesores y de las instituciones desde lugares a los auales no concurren; se analiza, juzga, se formulan propuestas, metodologías y hasta teońas sobre las decisiones, roles, pensamiento y conocimiento del profesor sin su participación real, adicionalmente, como consecuencia de la globalización y desarrollo de las comunicaciones, los sistemas de educación de los países están en constante ajuste, por lo que la profesión de profesor agudiza su vaivén al corte de las políticas de estado y la economía.

En este sentido, se puede observar que la educación y sus mayores impulsores, los profesores, se encuentran entre lo aspectos que mayor influencia tienen en la sociedad y en la economía, al igual que de variadas investigaciones, desafortunadamente 0 afortunadamente todos tienen que ver con esto, sin embargo, los directamente implicados, los profesores y estudiantes, poco o nada tienen de participación en las innovaciones o políticas de educación, a exención de participar en la ejecución de normas técnicas y administrativas que indican el camino a seguir, por lo cual su nivel de autonomía es muy bajo, quedando supeditado a las decisiones tomadas desde el exterior que como veremos más adelante afectan su imagen profesional.

\section{Acciones que afectan la profesión de ser profesor}

Moreno, et al. 2006, precisan que: "... al dirigir la mirada hacia la profesión y sus profesionales [los profesores] y ver auál es el posicionamiento que éstos tienen en la sociedad y sobre su propio quehacer [es] donde se evidencia la importancia de la reflexión sobre el saber pedagógico. Diversos estudios muestran que el problema está referido a la pérdida de su estatuto profesional y la consiguiente falta de identidad profesional, no solo porque objetivamente existen situaciones y condiciones que lo alejan de éste, sino también, por la pérdida de la legitimidad de la profesión, asociada a la pérdida de un saber institucionalizado como propio y exclusivo." (Moreno, et al. 2006: 29).

En relación con la perdida del estatuto profesional e identidad del profesor, se puede identificar que tal situación se desarrolla a partir de la concepción que se tiene y maneja sobre la enseñanza y el ¿Para qué? ¿Por qué?, y ¿Quiénes enseñan?, en cuanto a las dos primeras se puede decir de manera sucinta que para generar el desarrollo humano y porque de esta depende el desarrollo social, científico, tecnológico, económico y convivencial de un país, estos elementos se conjugan, 
regulan y equilibran dentro de los sistemas educativos. En este sentido la enseñanza es el punto nudear de ser profesor, generar procesos de aprendizaje, es el sello -no de calidad- sino personal de su profesión.

Pero entonces, ¿A qué se debe la perdida de identidad de la profesión de los profesores? de aquí se desprende que la enseñanza que el profesor ofrece tiene que ver básicamente con su formación profesional como profesor. Un profesor con formación profesional en la enseñanza que tiene un conocimiento y saber pedagógico y didáctico que puede estar en constante desarrollo y perfeccionamiento desde su actividad profesional de enseñante, esto asumiendo que la universidad en la cual se formó le ofreció la formación profesional. Tal vez uno de los aspectos por los que se ha perdido identidad es debido a que todos los profesores no tienen la formación como profesores, por lo tanto, tienen un conocimiento profesional muy válido sobre derecho, mediaina, antropología, sociología, agrónomo, ingeniería, etc., y necesario para la enseñanza, pero no suficiente como para ejercerla.

Los profesores que no tienen la formación como profesores, se enfrentan ante una realidad que solo han vivido como estudiantes aprendices durante su primaría, bachillerato y universidad, no obstante, se aventuran a la enseñanza, sin antes haber realizado una práctica y reflexiones sobre lo que significa ser profesor, enseñar, y desarrollar la relación y diferencias entre la naturaleza de un conocimiento disciplinar y el conocimiento pedagógico, didáctico, escolar. No se pueden negar que muchos de quienes ingresan en estás condiciones después de un tiempo adquieran los conocimientos y habilidades para posibilitar su quehacer pedagógico. En un principio van asumir la enseñanza de manera incierta, desprovistos del conocimiento del contenido didáctico, metodología estrategias. Varios superan la etapa y otros terminan por retirarse, mientras tanto muchos estudiantes constuyeron la idea de que los profesores no saben enseñar, induso pudieron haber desarrollado actitudes negativas hacia el aprendizaje de algunos conocimientos, al igual que haber construido concepciones distorsionadas sobre el conocimiento y quienes lo desarrollan.

Por el momento se encuentra que no hay límite para ser profesor, dado que se esta igualando la profesión de ser profesor a cualquier profesional. En este aspecto se vislumbra que la imagen social de la profesión de profesor, pierde identidad y legitimidad, al igual que propicia la degradación del estatuto profesional. No hay daridad sobre un conocimiento y saber como propio y exclusivo del profesor. Lo contrario ocurre si un profesor quiere ser médico, abogado, ingeniero sin tener conocimiento o saber sobre la profesión, esto sería algo imposible.

Ante esto, es posible señalar entonces que la profesión de profesor en realidad no es una es profesión o simplemente no esta darificada, por lo que se ha convertido es una opción laboral, sino de todas las profesiones si de la mayoría, todo profesional que quiera, crea o tenga la necesidad de ejercer como tal, lo puede hacer. 0 también puede ser considerada una profesión pero existen agentes externos que la vulneran, siendo lo peor no precisamente este hecho sino que los mismos profesionales profesores, muchas veces no tienen dara su profesión por lo que no hay suficientes pronunciamientos al respecto.

Desde este punto se darifica la idea de qué profesor están definiendo los encargados de fijar las políticas sobre las funciones, conocimiento y saber de los profesores, y de qué tipo de educación se esta buscando, y desde luego que también el tipo de ciudadano y ciudadana. Quedan entonces por el momento las preguntas sobre: ¿Qué hace que la educación sea vista al garete? ¿A qué o a quiénes beneficia este tipo de relación enseñanza profesión? y ¿Qué transformación requiere ésta situación?

Miremos una visión diferente, mediante una entrevista realizada a Helsinki a Jouni Valijaarvi, del Instituto de Investigación Educacional, al preguntarle el porqué de la calidad educativa finesa; lo primero que contestó, fue que lo fundamental para ellos es el valor que se les da a los profesores: 
"A diferencia de muchos países, la responsabilidad pedagógica recae en los profesores. Son ellos quienes deciden el sistema de calificación y el material que van a utilizar, en un ambiente muy individualista que apela más a un alto sentido de la ética que a controles estrictos"... "Esa independencia del maestro hace que la profesión sea muy popular. Tanto que apenas hay aupo para dos de diez personas que se presentan a las universidades para cursar los cuatro años que se requieren como mínimo para ser maestro". La entrevista se realiza a propósito de los resultados de la prueba PISA -Programme for International Student Assessment, Programa Internacional de Evaluación de Estudiantes- en la cual Finlandia es, de 57 países, de nuevo el primer país en calidad educativa (Hurtado, 2008).

Existe otro elemento que afecta negativamente la imagen de la profesión del profesor, este es de naturaleza económica, más que social y magisterial, que limita y delimitan desfigurando el ejercicio de la profesión al quitarle autonomía, al imponer y obligar a los profesor a cumplir con un porcentaje mínimo de estudiantes que deben ser promovidos al siguiente grado.

Por ejemplo, existe una norma que establece la obligación de promover el $95 \%$ de la población estudiantil al siguiente grado escolar, desde preescolar hasta el bachillerato, a través de este mecanismo el profesor debe hacer de lado los procesos de seguimiento de aprendizaje y comprensión que ha hecho a los estudiantes para dejar que la norma técnica decida por él.

En relación con esto lo que queda daro es que: primero los costos que representan para el estado la perdida de un año escolar por estudiante se ven reducidos (ya se esta pensando indusive que se debe promocionar el $\mathbf{1 0 0 \%}$ ), segundo, se desconocen los procesos pedagógicos, didácticos y evaluativos que siguen los profesores que buscan el aproximar el conocimiento al estudiante, para el desarrollo conceptual, actitudinal y procedimental, cuarto, no se pretende que se formen ciudadanos ilustrados, á́ticos, innovadores y propositivos (los niveles de exigencia son muy bajos, dado que los estudiantes conocen que van a ser promovidos estudien o no), quinto, con todo esto la función e imagen del profesor y su profesión queda una vez más en entredicho.

Al referente, Bustamante (2008), señala que las normas son elaboradas "desde el nivel jerárquico más alto hacia el nivel más bajo"; como cabe de esperar quienes establecen las normas, por lo general, son personas que poco o nada conocen sobre la educación, solo se centran en mirar los costos per cápita, las exigencias de la política extranjera e intereses personales.

En este mismo sentido, Carr (1993) pone de relieve que "aquellos que no son educadores profesionales -como los políticos, economistas, y empresarios- tenderán a interpretar y valorar la calidad en la enseñanza en términos de valores ajenos al proceso educativo. Desde sus perspectivas, la educación es vista como algo que sirve a propósitos extrínsecos, como el interés nacional, las necesidades económicas de la sociedad o las demandas del mercado de trabajo" ( $p$. 7).

Es así pues, como prácticamente no hay límites para ejercer como profesor, pero si existen límites de eficiencia escolar, sin tener en cuenta que la educación no puede ser vista como una fabrica de producción al estilo fordista, en la que los estudiantes son la materia prima y los profesores los obreros que simplemente tienen que cumplir ordenes sin necesidad de pensar 0 decidir. Como subraya, Torres (1994), al final, lo que de verdad importa son exdusivamente las notas, al igual que a los obreros y obreras los salarios. El producto y el procesos no merecen la pena, solo es importante el resultado extrínseco, el salario o las calificaciones escolares.

Detrás de esto se esconde que los estudiantes terminan con niveles de conocimiento muy bajo, desde luego que en este caso el profesor es visible, no obstante, se ve imposibilitado para exigir a los estudiantes a cumplir y ser responsable con su aprendizaje. En orden a esto, la responsabilidad 
sobre la educación, la enseñanza y aprendizaje de los futuros ciudadanos y ciudadanas, sigue siendo de los profesores, sean formados o no como profesionales de la educación, y así no sean quienes formulen las políticas de educación.

Esto es lo que Contreras (1999) plantea como pseudoparticipación, se concede autonomía escolar, pero los parámetros de las políticas ya están fijados, otras instancias han decidido y planificado todo, hasta las puertas de la escuela, los profesores actúan, pero sin capacidad de intervenir "hacia arriba" para transformar las condiciones en que se les deja "autónomos". Son sólo obedientes funcionarios que deben resolver los problemas en los que se encuentran, tal y como se los han definido y con los recursos que les han concedido. Su autonomía no es política, sino tan sólo de gestión.

Este mismo autor plantea que a los profesores se les aumenta su responsabilidad, pero sin aumentar su poder, más responsabilidad no significa más poder de cambiar las cosas, mayor responsabilidad supone un mayor deterioro de la profesión y un mayor rechazo social, al recibir todas las culpas por la incapacidad de hacerse cargo de todas las misiones sociales que se le encomiendan a la educación.

En el centro de toda este marco situacional de la profesión del profesor se encuentra el hecho de desconocer por parte de los entes administrativos y legislativos que existe un saber pedagógico que el es propios a las prácticas pedagógicas del profesor, que se construye, por lo menos, desde la formación inicial del profesor y que se consolidad con la experticia en el aula de dase, no desde un escritorio para legislar de acuerdo a las políticas económicas e ideológicas del momento, o desde el pleno de las investigaciones en educación que proponen teorías, innovaciones, sin reconocer el saber práctico, experiencial y contextual del profesor.

En cuanto a esto, cabe resaltar lo que plantea Marcia Prieto (1994), citada por Moreno, et al. 2006, sobre el saber pedagógico: "es el conjunto de conocimientos con estatus teórico y práctico que conforman un dominio de saber institucionalizado el cual configura la práctica de la enseñanza y la adecuación de la educación en una sociedad." Esto no quiere decir que con el saber pedagógico se van a resolver todo el problema sobre la degradación de la profesión del profesor, pero si puede ayudar a reconocer el estatus profesional del profesor.

Según esto, se evidencia la necesidad de dar participación al profesor, no solo en la gestión de las dases, curnículo, planes de estudio, sino también investigativa y legislativa, para que las normas y la teoría se acerquen a la dinámica escolar y no se conviertan, la una en empobrecimiento social y la otra en letra muerta.

Para terminar, Adorno y Becker (1994) aludiendo y entrelazando el escrito kantiano "¿Qué es la ilustración?", a la realidad de la educación actual, resaltan que: "Es realmente un fenómeno interesante de qué manera la educación para la minoría de edad domina el mundo hoy como ayer, aunque el tiempo de la ilustración está en marcha desde hace algún tiempo...".

\section{A manera de conclusión}

No se puede desconocer las capacidades de profesionales distintos a los de la educación pero tampoco se puede desconocer la necesidad de tener la formación pedagógica y didáctica para la enseñanza.

Hasta tanto no se reconozca la importancia de la educación para la sociedad, se seguirán observando situaciones como las expresadas en este escrito y continuaremos siendo un país con escaso desarrollo científico y tecnológico, con desigualdad social y violencia, que se inicia 
precisamente desde la educación, debido a la falta de exigencia académica, donde todo se vuelve fácil y sin sentido, ya que todo esta ganado desde el principio.

De no cambiar las cosas, continuará siendo responsabilidad de los profesores el fracaso o el éxito que tenga la educación en la sociedad, a expensas de las políticas económicas e ideológicas del estado.

\section{Bibliografía}

Adorno, T., y Becker. H. (1994). Educación para la mayoría de edad. Revista Colombiana de Psicología. (3), 11-18.

Bustamante, Z. (2008). El decreto 230 de 2002 y sus implicaciones en el desarrollo de una política de educación de calidad. Documento Secretaria de Educación de Bogotá.

Carr, W. (1989). Calidad de la enseñanza e investigación-acción. Sevilla: Díada.

Contreras, J. (1997). La autonomía del profesorado. Madrid: Morata.

Contreras, J. (1999). ¿Autonomía por decreto? Paradojas en la redefinición del trabajo del profesorado. Education Policy Analysis Archives. Vol 7 (17).

http: //epaa.asu.edu/epaa/v7n17.html

Hurtado, A. Maestro: Aguante y cállese. El Tiempo, Bogotá, 9 de septiembre de 2008.

Moreno, $\mathbf{N}$, et al. (2006). Tras las huellas del saber pedagógico. Universidad Pedagógica Nacional.

Torres, J. (1994). Contenidos interdisciplinares y relevantes. Cuademos de Pedagogía, Mayo (225), 19-24. 